\title{
Distribution of Basal Cell Carcinoma and Squamous Cell Carcinoma by Facial Esthetic Unit
}

\author{
Jung Hun Choi, Young Joon Kim, Hoon Kim, Sang Hyun Nam, Young Woong Choi \\ Department of Plastic and Reconstructive Surgery, Sanggye Paik Hospital, Inje University College of Medicine, Seoul, Korea
}

Background The most common cutaneous malignant masses are basal cell carcinoma (BCC) and squamous cell carcinoma. The predominant site of a malignant mass is the face, which has many opportunities to be exposed to ultraviolet radiation. However the predilection sites of malignant masses have been equivocal due to the use of general regions, rather than anatomical landmarks, in surveys. A facial esthetic unit is an anatomical site classified as an area of similar facial contour characteristics that can be distinguished from other areas. The purpose of this study is to determine widely accepted anatomical landmarks using the esthetic unit.

Methods We retrospectively analyzed 118 cases of malignant masses in our clinic from January 2005 to October 2012. We evaluated the patients' age, gender, and predilection site of the malignant mass by esthetic unit through pathology, medical records and patient photographs. We mapped the occurrence site of the malignant mass on schematic drawings of the esthetic units.

Results Most of the malignant masses were BCC. The ratio of males to females was 1:1.41. The frequent predilection site of a malignant mass was on the nasal unit (33.1\%), followed by the buccal unit (11.0\%). Primary closure was the most common method of repairing a surgical defect (38.9\%), followed by a local flap (35.5\%).

Conclusions This review described the relationship between clinical characteristics and esthetic units by proposing objective predilection sites for malignant masses, which can be used commonly as a framework in the study of malignant masses by unifying equivocal occurrence sites.

Keywords Carcinoma, basal cell / Carcinoma, squamous cell / Esthetics
Correspondence: Young Woong Choi Department of Plastic and Reconstructive Surgery, Sanggye Paik Hospital, Inje University College of Medicine, 1342 Dongil-ro, Nowon-gu, Seoul 139-707, Korea

Tel: +82-2-950-1048

Fax: +82-2-932-6373

E-mail:pshero2@naver.com

No potential conflict of interest relevant to this article was reported.

\section{INTRODUCTION}

The incidence of cutaneous malignant masses has increased gradually with changes in the social and medical environment [1]. Major cutaneous malignant masses including basal cell carcinoma (BCC), squamous cell carcinoma (SCC) and malignant melanoma (MM) result from multifactorial causes such as environmental and host factors [2]. In particular, ultraviolet radiation (UVR), an environmental factor, is estimated to be one of the most important risk factors [3]. In most reports, the general predilection site of a cutaneous malignant mass is the head and neck because there are many opportunities for these regions to be exposed to ultraviolet radiation [2-9]. However, the specific predilection site of a cutaneous malignant mass on the face has been ambiguous because the descriptions of the occurrence sites of such cutaneous malignant masses have differed in each study, which have used general sites, rather than anatomical landmarks. We believe that the use of unified common landmarks 
would aid in determining the predilection site of a cutaneous malignant mass. An esthetic unit, according to Gonzalez-Ulloa, is an anatomical site that is classified as an area of similar facial contour characteristics that can be distinguished from other areas [10]. These units were suitable for our study due to their definite boundaries. The purpose of this study was to determine widely accepted anatomical landmarks using the modified facial esthetic unit. We investigated the predilection sites of cutaneous malignant masses to confirm their objective anatomical distribution based on the modified facial esthetic units we defined.

\section{METHODS}

We retrospectively reviewed 116 patients who had surgical treatment due to a cutaneous malignant mass from January 2005 to October 2012 with in our department of reconstructive and plastic surgery. The clinical data included the patients' gender, age, predilection site and method of surgical treatment. To make the predilection site objective, two independent doctors who were not involved with the surgery classified the site of the cutaneous malignant mass according to the facial esthetic unit. A series of 5 photographs were made of the frontal view, oblique views ( $\mathrm{RH}$ right and LH left), and lateral views ( $\mathrm{RH}$ and $\mathrm{LH})$. We mapped the occurrence site of the cutaneous malignant mass on schematic drawings of the facial esthetic units without left and right distinctions. The results of this study were compared with those of other studies.

\section{Anatomical definition of the facial esthetic unit}

Referring to the facial aesthetic units suggested by GonzalezUlloa in 1954, the esthetic units were modified to create the following 12 units for this study. The forehead was divided into the frontal and temporal units by the pterion, the orbital area was divided into three parts: the orbital unit, supraorbital unit (including the eyebrows), and infraorbital unit between the nasojugal folds and the medial area of the zygoma. The cheek, was divided into three parts: the buccal unit around the soft, mobile anterior; the parotid-masseteric unit, which is the firm, mobile posterior region containing the parotid gland and the zygomatic unit, in which the zygomatic bone is located. The nasal unit, including the nose, is bordered by the philtrum and the nasolabial fold. The labial unit, including the lip, is bordered by the philtrum and the labiomental fold. The mental unit is bordered by the labiomental fold and menton. The auricular unit was defined as the periauricular region, including the ears (Fig. 1). Based on these units, we confirmed the distribution of the cutaneous malignant masses.

\section{RESULTS}

\section{Distribution of gender and age}

For the 118 cases, the most common cutaneous malignant mass was BCC (64.4\%), followed by SCC (32.2\%). The cases included 49 males and 69 females. The ratio of male to female patients was 1:1.38 in BCC and 1:1.71 in SCC. The age distribution ranged from 36 to 103 years old. The average age of the patients was 70.9 years at the time of diagnosis. The peak of the age-frequency distribution was the seventh decade in BCC, but it was the eighth decade in SCC.

\section{The anatomical site of cutaneous malignant masses by the modified facial esthetic unit}

The nasal unit was the predilection site for cutaneous malignant masses (33.1\%), followed by the buccal unit (11.0\%). The most

\section{Table 1. Anatomical site of cutaneous malignant masses by esthetic unit}

\begin{tabular}{lccccccc}
\hline Unit & BCC & SCC & MM & Ewing & BSCC & Muc & Total \\
\hline Frontal & $3(3.9)$ & $2(5.3)$ & $1(100)$ & $1(100)$ & $0(0)$ & $0(0)$ & $7(5.9)$ \\
Temporal & $4(5.3)$ & $5(13.2)$ & $0(0)$ & $0(0)$ & $0(0)$ & $0(0)$ & $9(7.6)$ \\
Auricular & $0(0)$ & $5(13.2)$ & $0(0)$ & $0(0)$ & $0(0)$ & $0(0)$ & $5(4.2)$ \\
Supraorbital & $3(3.9)$ & $2(5.3)$ & $0(0)$ & $0(0)$ & $1(100)$ & $0(0)$ & $0(5.1)$ \\
Orbital & $8(10.5)$ & $0(0)$ & $0(0)$ & $0(0)$ & $0(0)$ & $0(0)$ & $8(6.8)$ \\
Infraorbital & $4(5.3)$ & $2(5.3)$ & $0(0)$ & $0(0)$ & $0(0)$ & $1(100)$ & $7(5.9)$ \\
Nasal & $37(48.7)$ & $2(5.3)$ & $0(0)$ & $0(0)$ & $0(0)$ & $0(0)$ & $39(33.1)$ \\
Zygomatic & $2(2.6)$ & $4(10.5)$ & $0(0)$ & $0(0)$ & $0(0)$ & $0(0)$ & $6(5.1)$ \\
Buccal & $5(6.6)$ & $8(21.1)$ & $0(0)$ & $0(0)$ & $0(0)$ & $0(0)$ & $13(11.0)$ \\
Labial & $7(9.3)$ & $1(2.6)$ & $0(0)$ & $0(0)$ & $0(0)$ & $0(0)$ & $8(6.8)$ \\
Parotid-masseteric & $1(1.3)$ & $7(18.4)$ & $0(0)$ & $0(0)$ & $0(0)$ & $0(0)$ & $0(0)$ \\
Mental & $2(2.6)$ & $0(0)$ & $0(0)$ & $0(0)$ & $0(0)$ & $1(0.8)$ & $2(1.7)$ \\
Total & $76(64.4)$ & $38(32.2)$ & $1(0.8)$ & $1(0.8)$ & $1(0.8)$ & $1(0.8)$ & $118(100)$ \\
\hline
\end{tabular}

Values are presented as number (\%).

BCC, basal cell carcinoma; SCC, squamous cell carcinoma; MM, malignant melanoma; Ewing, Ewing's sarcoma; BSCC, basosquamous cell carcinoma; Muc, mucinous carcinoma. 
common location of the occurrence of BCC was the nasal unit (48.7\%), followed by the orbital unit (10.5\%) in our study. SCC occurred most commonly on the buccal unit $(21.1 \%)$, followed by the parotid-masseteric unit (18.4\%) (Table 1). The predilection sites for BCC and SCC were mapped on schematic drawings of the facial esthetic units (Fig. 2).

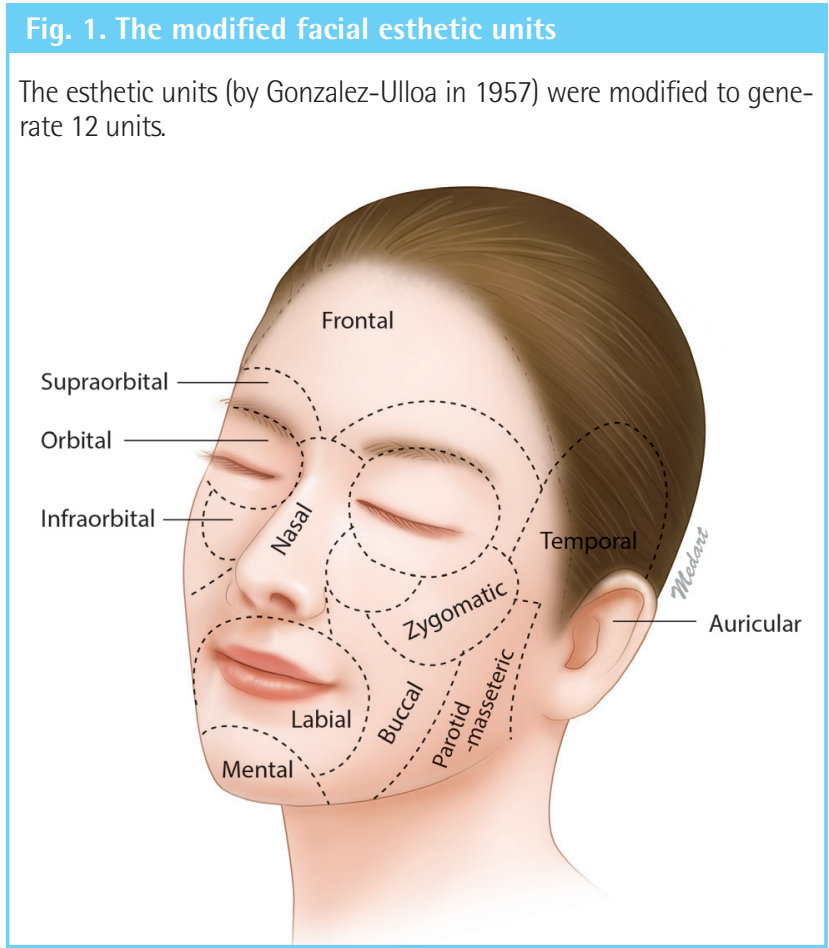

\section{Surgical treatment}

Primary closure was the most common method for repairing any surgical defect (38.9\%), followed by a local flap (35.5\%). The coverage of BCC was performed most often by primary closure (39.5\%), followed by a local flap (34.2\%). The surgical defect from SCC was most commonly repaired by a local flap (39.5\%), followed by primary closure (34.2\%).

\section{DISCUSSION}

The incidence of cutaneous malignant masses has increased rapidly worldwide over the last decade due to extended life spans and social and medical changes $[4,11]$. The rising incidence rate is most likely due to a combination of increased ultraviolet radiation, increased outdoor activities, changes in clothing style, and ozone depletion [12]. In a recent study, a cutaneous malignant mass was the most commonly diagnosed cancer in the United States, with more than 1 million Americans diagnosed with skin cancer each year [1]. Additionally, in Korea, the incidence of cutaneous malignant masses was reported to be approximately $4 \%$ of all malignant masses and this incidence has been increasing steadily [5]. Cutaneous malignant masses have been classified as non-melanoma skin cancer and MM. The most common cutaneous malignant masses are BCC and squamous cell carcinoma. In this study, the majority of cutaneous malignant masses were BCC (64.4\%) and SCC (32.2\%).

The incidence of cutaneous malignant masses increases with

\section{Fig. 2. Distribution of basal cell carcinoma on facial esthetic units}

(A) The predilection sites for basal cell carcinoma were mapped on schematic drawings of the facial esthetic units (red triangle). (B) The predilection sites for squamous cell carcinoma were mapped on schematic drawings of the facial esthetic units (blue square).
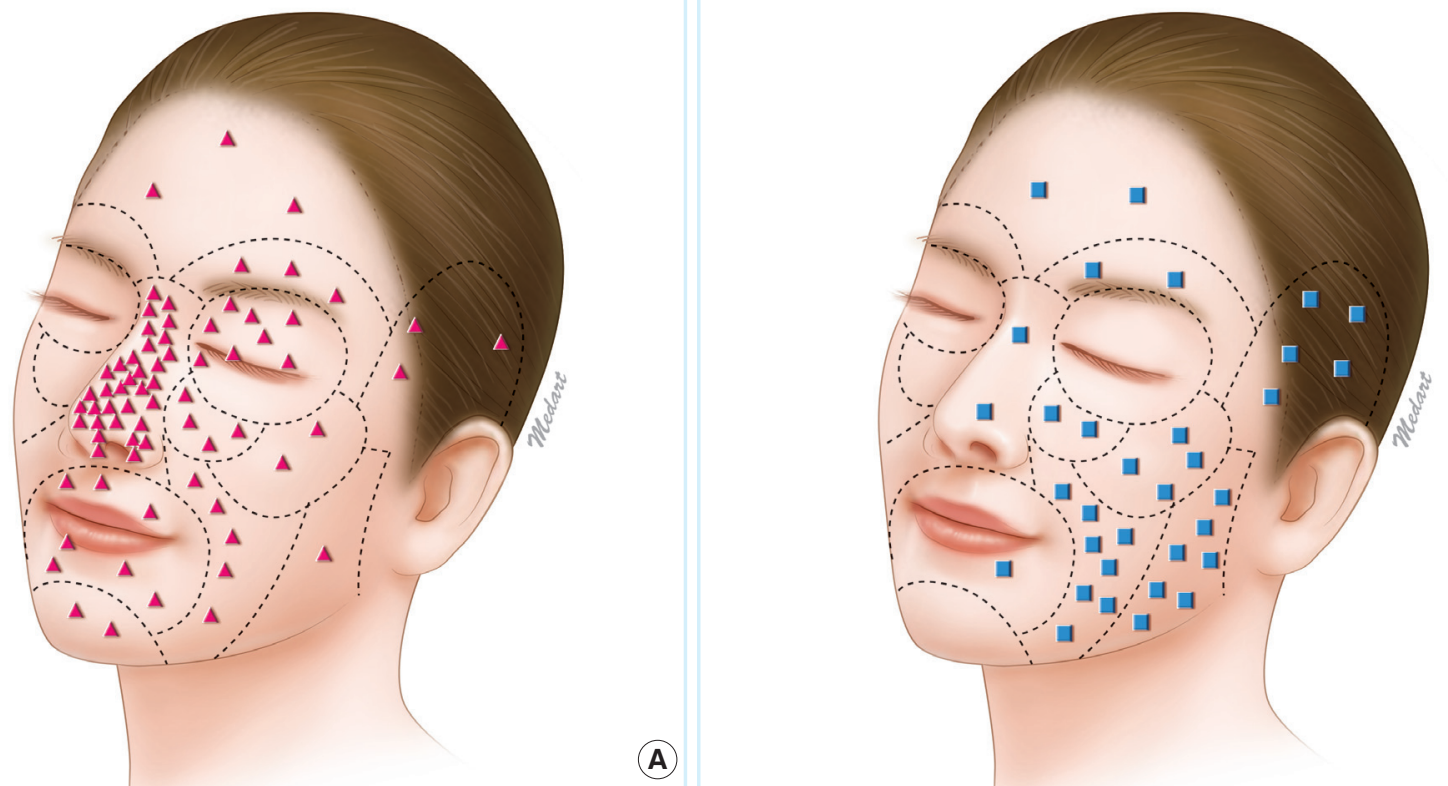
age due to the patients' increased susceptibility and the cumulative dose of UVR [13]. In this study, the most frequent age group was the seventh decade, and patients over 40 years of age accounted for $95.8 \%$ of our population. The average age of the patients was 70.9 years. The incidence of cutaneous malignant masses has been shown to be higher in males than in females due to their more extensive exposure to sunlight [14]. However, in recent studies, Lee et al. [15] reported a ratio of 1.19:1, and Seo et al. [16] reported a ratio of 1.1:1, showing no significant differences by gender. Shin et al. [5], Yoon et al. [17], and Jeong et al. [18] have reported ratios of $0.94: 1,0.94: 1$, and $0.98: 1$, respectively, thus indicating a higher incidence in females. In this study, females were more frequently affected, with a male to female ratio of $0.72: 1$. In other studies, the reasons for the higher female incidence were determined to be the extended life expectancy of women and their increased chance of exposure to sunlight [19].

BCC and SCC are usually found in sun-exposed areas, especially the head and neck regions $[20,21]$. Many studies have demonstrated that the predilection site of cutaneous malignant masses is in the face. Bart et al. [22] noted a higher rate of occurrence and recurrence in masses located on the ear and lips. Buettner and Raasch [23] estimated the body site-specific incidence rate for cutaneous malignant masses and reported that it was most commonly found in the lip, orbit and naso-labial areas. In the domestic literature, Kim et al. [8] reported that BCC had occurred on the cheek, followed by the periorbital area and nose; Jung and Kim [3] reported that, BCC was found most frequently on the nose (38.4\%). Kim et al. [9] also reported the nose as being the most common region for BCC (47.3\%). In the case of squamous cell carcinoma, Jung and Kim [3] reported that it occurred most commonly on the lower lip (41\%), followed by the cheek (23.2\%), where as Kim et al. [9] reported that it occurred on the lower lip (26.2\%) and cheek (21.4\%). However, the predilection site of each study was ambiguous because the descriptions of the cutaneous malignant mass sites differed due to the use of general terminology, rather than anatomical terminology. In addition, the operator subjectively assigned the actual location of the mass. If a different observer performed the operation, different results would have been obtained. These studies, therefore, $c$ annot be compared with each other.

Several studies separated the occurrence region based on the anatomical site. The H-zone (i.e., nasolabial fold, nasal alar, orbital area and auricular area) was found to be the most common site of recurrence in BCC [7]. Mora and Robins [6] found that $\mathrm{BCC}$ growing in the triangle of the face (formed by lines drawn from the outer canthi to the philtrum of the lip) tends to be more invasive, more destructive and more recurrent than that in other sites. However, they also did not obviously divide the boundary.
In this study, we used facial esthetic units to avoid the confusion of communication that occurred by equivocal boundaries in each previous study. Gonzalez-Ulloa [10] is defined the facial esthetic unit as an anatomical unit that separates similar regions of contour characteristics, such as color, texture, thickness, elasticity and sebaceous gland density. Therefore, resection or reconstruction within this unit can achieve the best aesthetic results [7]. This study, used facial esthetic units that included obvious and detailed boundaries to objectively analyze, the predilection sites of cutaneous malignant masses, and the boundaries were thus easier to distinguish. In this study, the nasal unit was the predilection site of cutaneous malignant masses (33.1\%), followed by the buccal unit (11.0\%). We drew a similar conclusion as those in other studies. In particular, the predilection site is similarly concentrated in the H-zone explained above, but more studies about whether there is a match between the predilection site and recurrence site will be needed. In addition our results could support the hypothesis that exposure to UVR appears to be the most important risk factor based on the high incidence on the protrusion sites of the face.

As a result of this study, we confirmed the relationship between the clinical characteristics and the facial esthetic unit. The predilection site of a cutaneous malignant mass can be suggested objectively and thus used commonly in a study of cutaneous malignant masses as a framework to unify the equivocal occurrence site. In addition, distinguishing cutaneous malignant masses according to the facial esthetic unit can be used as a reference plane during reconstruction.

However, the results of this study are not likely to represent the prevalence and the most common site because this study is a single center analysis with a relatively small number of patients. In the future, statistical results from other hospitals should be studied together to form a general consensus.

\section{REFERENCES}

1. Glanz K, Schoenfeld ER, Steffen A. A randomized trial of tailored skin cancer prevention messages for adults: Project SCAPE. Am J Public Health 2010;100:735-41.

2. Hwang JI, Kim HS, Park H, et al. A statistical survey of major cutaneous malignant tumors for the last 10 years (20002010, North-east Gyeonggido Province). Korean J Dermatol 2011;49:97-105.

3. Jung YH, Kim SS. A clinical study in malignant skin tumors. J Korean Soc Plast Reconstr Surg 1982;9:377-88.

4. Hong H, Ji JH, Choi EH. A clinical observation of cutaneous malignant tumors and premalignant lesions in gangwon province over 10 years (1999-2008). Korean J Dermatol 
2012;50:95-100.

5. Shin JH, Cho S, Whang KK, et al. An epidemiologic analysis of cutaneous malignant tumors over 15 years (1984-1998). Korean J Dermatol 1999;37:1743-51.

6. Mora RG, Robins P. Basal-cell carcinomas in the center of the face: special diagnostic, prognostic, and therapeutic considerations. J Dermatol Surg Oncol 1978;4:315-21.

7. Kim JW, Oh CH, Kim IH. Distribution of histologic subtypes of basal cell carcinoma by facial aesthetic unit and subunit. Korean J Dermatol 2000;38:31-7.

8. Kim HR, $\mathrm{NaCH}$, Shin BS, et al. A statistical study of cutaneous basal cell carcinoma and squamous cell carcinoma in Gwangju City and Chonnam Province (2006-2010). Korean J Dermatol 2011;49:1073-8.

9. Kim YP, Chun IK, Lee HH. A 10 year period (1968-1977) of clinical observation of cutaneous malignant tumors. Korean J Dermatol 1978;16:19-29.

10. Gonzalez-Ulloa M. Restoration of the face covering by means of selected skin in regional aesthetic units. $\mathrm{Br} \mathrm{J}$ Plast Surg 1956;9:212-21.

11. Armstrong BK, Kricker A. Skin cancer. Dermatol Clin 1995; 13:583-94.

12. Gloster HM Jr, Brodland DG. The epidemiology of skin cancer. Dermatol Surg 1996;22:217-26.

13. Giles GG, Marks R, Foley P. Incidence of non-melanocytic skin cancer treated in Australia. Br Med J (Clin Res Ed)
1988;296:13-7.

14. Vana J. Epidemiology. In: Helm F, editor. Cancer dermatology. 1st ed. Philadelphia: Lea \& Febiger; 1979. p.3-15.

15. Lee YJ, Seo SJ, Kim MN, et al. A 10 year period (1984-1993) of clinical observation of cutaneous malignant tumors. Korean J Dermatol 1995;33:679-85.

16. Seo JJ, Won YH, Kim SJ, et al. A clinical observation of cutaneous malignant tumors over 10 years (1987-1996, Chonnam Province ). Korean J Dermatol 1998;36:812-9.

17. Yoon TJ, Kim HS, Oh CW, et al. A clinical investigation of cutaneous malignant tumors in western Gyeongnam Province. Korean J Dermatol 1997;35:956-62.

18. Jeong KB, Kim HC, Shin DH, et al. A clinical observation of cutaneous premalignant and malignant tumors. Korean J Dermatol 2002;40:924-31.

19. Cho KH, Lee YS. A clinical observation of cutaneous malignant tumors. Korean J Dermatol 1984;22:394-403.

20. Narayanan DL, Saladi RN, Fox JL. Ultraviolet radiation and skin cancer. Int J Dermatol 2010;49:978-86.

21. Randle HW. Basal cell carcinoma. Identification and treatment of the high-risk patient. Dermatol Surg 1996;22:255-61.

22. Bart RS, Schrager D, Kopf AW, et al. Scalpel excision of basal cell carcinomas. Arch Dermatol 1978;114:739-42.

23. Buettner PG, Raasch BA. Incidence rates of skin cancer in Townsville, Australia. Int J Cancer 1998;78:587-93. 